\title{
Finite Element Simulation of Water Shock Tube Used for Dynamic Calibration of Underwater Shock Wave Pressure Sensor
}

\author{
Chundong Xu, Tingwei Gu, and Deren Kong
}

School of Mechanical Engineering, Nanjing University of Science and Technology, Nanjing, China

realngwei@163.com

Keywords: piezoelectric pressure sensor; dynamic calibration; water shock tube; Autodyn finite element simulation software

\begin{abstract}
In this paper, the characteristics of explosion shock wave in the water shock tube are studied by means of finite element simulation analysis. The principle of the comparison calibration method based on the pre-pressure water shock tube device is introduced, and the propagation rule of the explosion shock wave in the infinite water area and the water shock tube is simulated by Autodyn finite element software. The simulation results are compared with the empirical formula of the underwater explosion shock wave, and the corresponding similarity analysis of the shock wave pressure waveform is performed. The research results show that the water shock tube can generate a quasi- $\delta$ pulse signal with larger peak pressure at the end surface under the condition that the explosive mass is very small. In addition, when the length-diameter (L/D) ratio of the water shock tube is greater than 16 , the quasi- $\delta$ pulse signal can be guaranteed to be a stable plane wave and has higher similarity with the shock wave pressure under the actual underwater explosion condition.
\end{abstract}

\section{Introduction}

The underwater shock wave pressure is a significant technical index to evaluate the damage effect of various underwater weapon systems and also a major reference for the design of underwater armor and warship protection [1-3]. The most commonly used sensors are the tourmaline ICP (integrated circuits piezoelectric) underwater blast sensors, which are developed by the Naval Surface Weapons Center and produced by PCB company [4]. Piezoelectric pressure sensor has good dynamic characteristic and its natural frequency is up to several hundreds $\mathrm{kHz}$ [5]. However, the lowfrequency characteristic of the piezoelectric sensor is poor and it is difficult to obtain the sensor sensitivity accurately by static calibration [6]. Therefore, the dynamic calibration is necessary. Commonly used dynamic pressure calibration devices are sinusoidal pressure generator, shock tube and drop-weight device [7-9]. The sinusoidal pressure generator is an ideal dynamic pressure calibration device, and the frequency response of the sensor system can be accurately obtained through it. However, limited by the rotational speed of the motor, the frequency band and amplitude range of the pressure signal produced by the sinusoidal pressure generator are all small. Therefore, this calibration device is usually only applicable to periodic low-scale dynamic pressure calibration. The high frequency characteristics of the piezoelectric sensor can be easily obtained by the shock tube. However, due to the short duration of the step pressure, the low frequency characteristics can not be calibrated effectively. In addition, the pressure amplitude of the shock tube is low, and the dynamic calibration of the high pressure sensor can not be realized. Although the drop-weight device can produce a half sinusoidal pressure signal with an amplitude of several hundred MPa, the pulse width of the signal is usually a few milliseconds, and the frequency range of the pressure signal is only within $1 \mathrm{kHz}$. Therefore, the drop-weight device is usually used to calibrate the sensor sensitivity, but can not realize the dynamic calibration of the underwater shock wave pressure measurement system. In summary, the above three calibration devices can not simulate the actual working conditions of underwater shock wave pressure sensors, and the reliability of calibration results is not high.

In order to solve the above problems, the relevant scholars have used the spherical TNT 
explosives to calibrate the underwater shock wave pressure measurement system [10]. The method obtains the theoretical pressure value according to the calculation formula summed up by Cole and applies it to the sensitivity calibration of the measurement system. Because of the complexity of underwater explosion, the uncertainty of the pressure produced by the spherical TNT explosives and the standardization problem of the calibration experiment, the theoretical calculation pressure is quite different from the actual situation, and the calibration accuracy is hard to be guaranteed. Zhu Mingwu [11] proposed the calibration method based on the pre-pressure water shock tube, which can produce a quasi- $\delta$ pressure pulse for the dynamic calibration of the underwater shock wave pressure measurement system. This method has achieved good experimental results in the calibration practice and obtained the transfer function of the measurement system. In the dynamic calibration of the water shock tube, the scholars [12-14] are mainly based on the engineering experience, but do not study the propagation characteristics of shock wave pressure in the water shock tube and the related influence factors of the pressure pulse.

The actual underwater explosion usually does not appear the phenomenon of the wall reflection in a certain propagation distance. In this case, it can be considered that the explosion shock wave propagates in the infinite water area. However, when the spherical explosive in the water shock tube is detonated, the generated spherical shock waves are reflected continuously on the tube wall, which causes the pressure field in the water shock tube to be very complex. In order to ensure that the water shock tube can produce a shock wave similar to the actual underwater explosion, and provide an effective excitation source for the dynamic calibration of the pressure measurement system, it is necessary to study the characteristics of the pressure field in the water shock tube and the influence factors of the quasi- $\delta$ pressure pulse.

In view of the above problems, this paper uses the Autodyn finite element software to simulate the explosion process of the spherical TNT explosives in the infinite water area and the water shock tube. According to the simulation results, the formation law of the plane wave in the water shock tube, the similarity of the quasi- $\delta$ pulse and the shock wave pressure in the infinite water area, and the relationship between the quasi- $\delta$ pressure pulse and TNT explosive charge and length diameter ratio of the water shock tube are analyzed, and corresponding conclusions are drawn.

\section{Calibration principle and explosion theory}

\subsection{Calibration principle of pre-pressure water shock tube}

The overall structure of the pre-pressure water shock tube device is shown in Figure 1. The main body of the device is a high pressure-resisting thick-wall tube, which is filled with water. An ignition head is placed in the geometric center of the tube and can be used for detonating explosives. The calibrated sensor and the reference sensor are symmetrically installed at the two end faces of the water shock tube, and the pre-pressure device is connected to the tube by a special threaded joint. In actual dynamic calibration, a static pressure is firstly applied to the shock tube by the pre-pressure device, then the ignition head in the center of the tube is started, and the spherical explosive is detonated to produce a quasi- $\delta$ pressure pulse, which is similar to the underwater explosion shock wave and can be used as the excitation source signal.

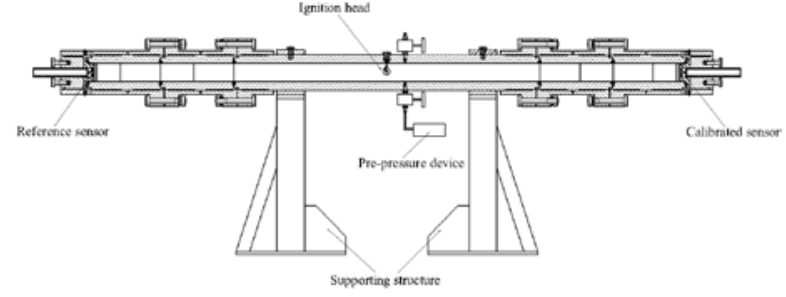

Figure 1. Overall structure of the pre-pressure water shock tube device.

As shown in Figure 2, when using the water shock tube for dynamic calibration, the reference pressure monitor system and the underwater shock wave pressure measurement system should be set up. 


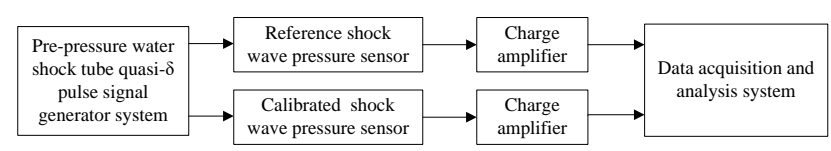

Figure 2. Reference pressure monitor system and underwater shock wave pressure measurement system.

The reference pressure monitor system for dynamic calibration should have good dynamic characteristics. It is necessary to ensure that its effective frequency range can cover the calibrated measurement system, and that it has a flat amplitude frequency characteristic in the effective frequency range. Under this premise, the reference pressure monitor system is calibrated by a highprecision piston pressure gauge and its value is traced to the national primary standard (mass standard and length standard). The calibrated shock wave pressure measurement system and the reference pressure monitor system are symmetrically arranged at the two end faces of the water shock tube, and the pressure amplitude and the rise time of the quasi- $\delta$ pressure pulse signal are changed by adjusting the mass of the spherical TNT explosive and the length of the water shock tube. Taking the pressure measured by the reference monitor system as the standard excitation signal and the pressure measured by the calibrated measurement system as the response signal, the transfer characteristic function of the calibrated measurement system can be obtained, and then the dynamic calibration of the underwater shock wave pressure measurement system can be realized.

\subsection{Shock wave theory of underwater explosion}

When explosives are detonated in water, a strong disturbance is generated. The disturbance is propagated in the form of compressed waves in the form of the water and produces a pressure jump in several microseconds. Then the pressure in the water attenuates in an approximate exponential law, and the duration of the attenuation is not more than a few milliseconds. The empirical formula of underwater shock wave pressure is obtained mainly from the shock wave similarity law and underwater explosion test data. Different formulas are obtained under different physical assumptions, explosives characteristics and test data, so their application scope is also different. The most classical empirical formula is proposed by Cole [15], which includes the mathematical relationship between the peak pressure and the distance to the explosion center and the pressure attenuates with time after the arrival of the shock wave.

$$
\left\{\begin{array}{l}
p_{m}=k\left(\frac{W^{\frac{1}{3}}}{R}\right)^{\alpha} \\
p=p_{m} e^{-t / \theta}
\end{array}\right.
$$

where $p_{m}$ is the peak pressure of underwater shock wave; $\mathrm{W}$ is the explosive charge; $\mathrm{R}$ is the distance to the explosion center; $\theta$ is the time attenuation constant, and $\theta=\frac{l}{k} W^{\frac{1}{3}}\left(\frac{W^{\frac{1}{3}}}{R}\right)^{\beta-\alpha}$; and $\alpha, \beta, k$, $l$ are empirical coefficients. For TNT explosives, $\alpha=1.13, \beta=0.89, k=52.27 \mathrm{MPa}, l=5768 \mathrm{Ns} / \mathrm{m}^{2}$.

\section{Finite element simulation}

\subsection{Simulation model}

To improve the simulation precision, it is necessary to ensure that the mesh size of the finite element model is small enough. However, if the grid of the finite element model is too small, it will take a long time to simulate. Therefore, the model of the explosion center can be established separately to simulate the detonation of the spherical TNT explosive in water, and then the simulation results can be introduced into the infinite water area or the water shock tube simulation model. Thus, the subsequent simulation of underwater shock wave pressure propagation can be carried out quickly and efficiently.

As shown in Figure 3, the two-dimensional (2D) explosion center quarter model in the infinite water area is established. The water medium and TNT explosive are meshed by the Euler method, 
and the mesh size is $0.05 \mathrm{~mm}$. The TNT explosive is spherical with a radius of $40 \mathrm{~mm}$ and a mass of $437 \mathrm{~g}$, and the initiation mode is point initiation. The length of the water area in the explosion center model is $100 \mathrm{~mm}$. To ensure that the underwater shock wave pressure can propagate outward without reflection in the water medium, the outflow boundary condition is adopted.

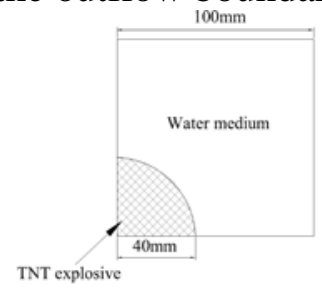

Figure 3. 2D explosion center quarter model in the infinite water area.

The 2D simplified model of the infinite water area is shown in Figure 4, and the simulation model is a sphere area with a radius of $1600 \mathrm{~mm}$, and the mesh size is $5 \mathrm{~mm}$. The outflow boundary condition is applied at the edge of the model, so that the shock wave pressure can propagate outward without reflection, thus simulating the actual underwater explosion conditions.

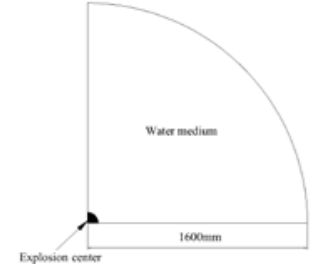

Figure 4. 2D simplified quarter model of the infinite water area.

Unlike in the infinite water area, the peak pressure decays slowly when the shock wave propagates in the water shock tube, so the explosive mass can be greatly reduced. In the simulation of the water shock tube, the radius of the spherical TNT explosive is set to $4 \mathrm{~mm}$ in the explosion center model, that is, the explosive mass is $0.437 \mathrm{~g}$. The $2 \mathrm{D}$ simplified quarter model of the water shock tube is shown in Figure 5. The water shock tube is meshed by the Lagrange method, the inner diameter is $100 \mathrm{~mm}$, the outer diameter is $200 \mathrm{~mm}$, and the length of the inner cavity is $3200 \mathrm{~mm}$. The water medium in the cavity is meshed by the Euler method, and the mesh size is $5 \mathrm{~mm}$, and the fluid-solid coupling method is automatic coupling.

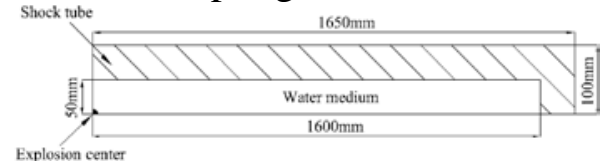

Figure 5. 2D simplified quarter model of the water shock tube.

The material of the TNT explosive, water medium and water shock tube can be directly loaded from the material library of the Autodyn software. The state equation of TNT explosive and water medium are JWL (Jones-Wilkins-Lee) model and Polynomial model respectively. The material of the water shock tube is AISI 4340 steel, and its strength model is Johnson-Cook model.

\subsection{Monitor points arrangement}

To understand the pressure distribution in the water shock tube, two pressure measurement points are set at every $100 \mathrm{~mm}$ in the propagation direction of the explosion shock wave, which can be used to monitor the pressure on the center and circumference of each cross section of the water shock tube. Similarly, multiple pressure measurement points are set at the same distance interval in the infinite water area simulation model. Figure 6 and Figure 7 show the distribution of pressure monitor points and the pressure cloud of explosion shock waves in the infinite water area and the water shock tube respectively. It can be seen from the figures that the explosion shock wave propagates mainly in the form of spherical waves in the infinite water area and propagates mainly in the form of plane waves in the water shock tube after a period of explosive detonation. 


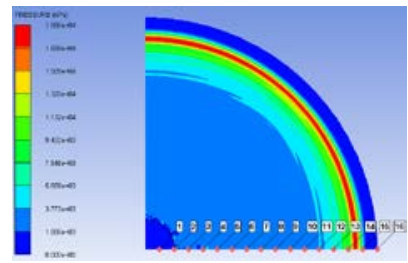

Figure 6. Distribution of pressure monitor points and the pressure cloud of explosion shock waves in the infinite water area.

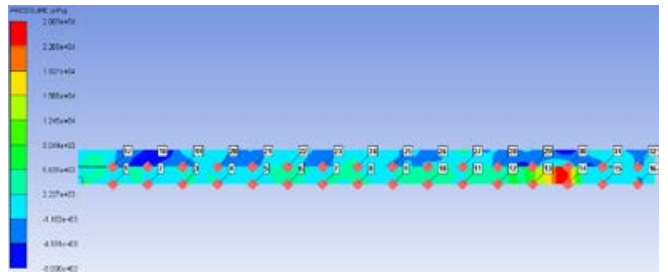

Figure 7. Distribution of pressure monitor points and the pressure cloud of explosion shock waves in the water shock tube.

\section{Results and discussion}

\subsection{Selecting a Template Waveform analysis of underwater shock wave}

The explosion shock wave pressure curves at the monitor points 5 10 in the infinite water area are shown in Figure 8, and the pressure waveforms are consistent with the classical empirical formula proposed by Cole. When the wave front of the underwater explosion shock wave propagates to the monitor point, the pressure amplitude first rises rapidly, and then attenuates according to the exponential rule. The rise time of the shock wave pressure is prolonged with the increase of the propagation distance, while the peak value is inversely proportional to the distance.

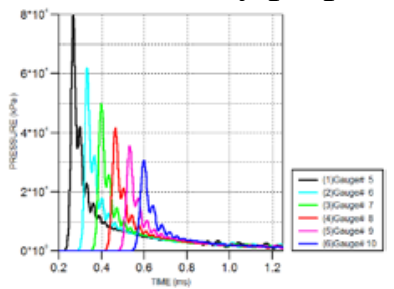

Figure 8. Explosion shock wave pressure curves at the monitor points $5 \sim 10$ in the infinite water area.

The comparison between the results of the finite element simulation and the classical empirical formula is shown in Table 1.

Table 1. Peak value and time attenuation constant of the explosion shock wave at the monitor points 5 10 obtained by finite element simulation and empirical formula respectively.

\begin{tabular}{|c|c|c|c|c|c|c|}
\hline $\begin{array}{c}\text { Monitor } \\
\text { point }\end{array}$ & $\begin{array}{c}p_{m} \text { obtained } \\
\text { by } \\
\text { Autodyn } \\
\mathbf{( M P a )}\end{array}$ & $\begin{array}{c}p_{m} \text { obtained } \\
\mathbf{b y} \\
\mathbf{C o l e} \\
\mathbf{( M P a )}\end{array}$ & $\begin{array}{c}\text { Difference } \\
\text { percentage of } \\
p_{m}\end{array}$ & $\begin{array}{c}\theta \\
\text { obtained by } \\
\text { Autodyn } \\
\mathbf{( \mu \mathbf { s } )}\end{array}$ & $\begin{array}{c}\theta \\
\text { obtained by } \\
\text { Cole } \\
(\boldsymbol{\mu s})\end{array}$ & $\begin{array}{c}\text { Difference } \\
\text { percentage of } \\
\theta\end{array}$ \\
\hline 5 & 79.87 & 83.75 & $4.63 \%$ & 56.28 & 75.76 & $25.71 \%$ \\
\hline 6 & 61.92 & 68.16 & $9.15 \%$ & 62.64 & 79.15 & $20.86 \%$ \\
\hline 7 & 49.88 & 57.26 & $12.89 \%$ & 65.95 & 82.13 & $19.70 \%$ \\
\hline 8 & 41.57 & 49.24 & $15.58 \%$ & 69.01 & 84.81 & $18.63 \%$ \\
\hline 9 & 35.37 & 43.11 & $17.95 \%$ & 75.29 & 87.24 & $13.69 \%$ \\
\hline 10 & 30.60 & 38.27 & $20.04 \%$ & 78.57 & 89.47 & $12.19 \%$ \\
\hline
\end{tabular}

It can be seen from Table 1 that there is a certain difference between the simulation results of the Autodyn software and the calculation results of the empirical formula, but it is not more than $25 \%$ in general, which can meet the requirements of the engineering applications. Therefore, using the 
Autodyn finite element software to simulate the underwater explosion has high credibility.

The shock wave pressure curves on the axis of the water shock tube is shown in Figure 9. Different from the shock wave pressure curves in the infinite water area, the shock wave pressure in water shock tube has obvious pressure disturbance before the arrival of the wave front. After detonating the TNT explosive, the shock wave will propagate through the water medium and the water shock tube cavity. Because the propagation velocity of the shock wave in the water is lower than that in the shock tube, the shock wave in the metal cavity will propagate to the end face of the shock tube first and produce a certain pressure disturbance in the vicinity. The explosion will also cause the rapid flow of water medium in the shock tube and the continuous vibration of the mechanical device, which leads to the presence of the residual pressure disturbance after the shock wave pressure is reflected away from the end face of the shock tube. In addition, when the propagation distance of the shock wave is short, for example, the shock waves at the monitor points 2 and 3 are affected by the reflection of the surrounding wall, and the waveforms are very chaotic. When the shock wave propagates a certain distance, such as the pressure measurement points 8 and 10 , the pressure curve gradually accords with the distribution rule of the typical shock wave pressure curve. At $1.046 \mathrm{~ms}$, the shock wave propagates to the two end faces of the water shock tube, and the peak pressure changes to about two times of the original value after the reflection (such as the pressure measurement point 16), and then the shock wave begins to propagate in the opposite direction (such as the pressure measurement point 13).

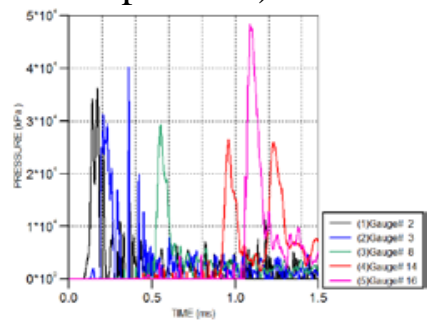

Figure 9. Shock wave pressure curves on the axis of the water shock tube.

The explosion shock wave pressure curve Under the actual working conditions is shown in Figure 7 , and the peak pressure decreases rapidly with the increase of the distance from the explosion center. Figure 9 shows that the peak pressure of the explosion shock wave in the water shock tube decays slowly, and remains almost unchanged during the propagation process. It is assumed that the peak pressure of the shock waves in the infinite water area and the water shock tube are $P_{1}$ and $P_{2}$ respectively, and the comparison results between the two are shown in Table 2.

Table 2. Comparison results between the peak pressure of the shock waves in the infinite water area and the water shock tube.

\begin{tabular}{|c|c|c|c|c|c|c|c|c|}
\hline $\begin{array}{c}\text { Propagation } \\
\text { distance } \\
\text { (mm) }\end{array}$ & 200 & 400 & 600 & 800 & 1000 & 1200 & 1400 & 1600 \\
\hline$P_{1}$ (MPa) & 299.58 & 109.68 & 61.92 & 41.57 & 30.60 & 23.91 & 19.44 & 16.81 \\
\hline$P_{2}$ (MPa) & 36.10 & 29.99 & 30.44 & 29.36 & 25.90 & 26.29 & 26.40 & 48.35 \\
\hline
\end{tabular}

Although the TNT explosives in the infinite water area is 1000 times the mass of that in the water shock tube, the peak pressure in the water shock tube has gradually exceeded that in the infinite water area with the increase of the propagation distance, and the pressure attenuation is slow and eventually tends to stability. When the shock waves reflect at the two end faces of the water shock tube, the peak pressure will increase to about two times as much as the original value. Therefore, the water shock tube can produce much greater shock wave pressure than the actual underwater working condition with very little mass of explosives, which can effectively save the cost of the calibration test.

\subsection{Formation rule of plane wave in water shock tube}

The quasi- $\delta$ signal used for dynamic calibration in the water shock tube is usually required as a 
plane wave. In the early stage of the explosion, under the influence of the surrounding wall reflection, the water shock tube not only has the spherical waves from the explosion center, but also various reflection waves in different states. These two kinds of shock waves superimpose each other and propagate a certain distance along the axial direction of the water shock tube before gradually forming a stable plane wave.

When a stable plane wave is formed in the water shock tube, the pressure on the wavefront should be consistent with the waveform and the peak value. Compare the most typical pressures on the center and circumference of the cross section of the water shock tube, that is, the pressures monitored by the two measurement points at the same propagation distance in Figure 7, and the comparison results are shown in Figure 10.

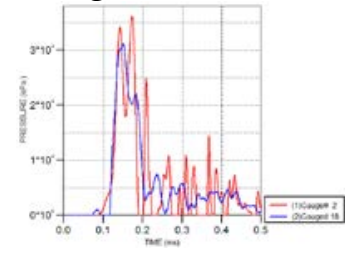

(a)

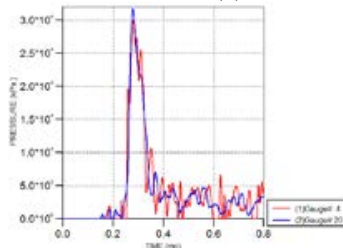

(c)

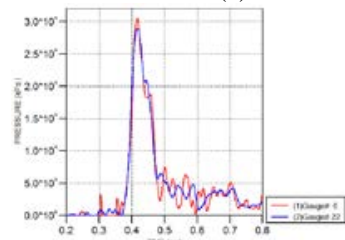

(e)

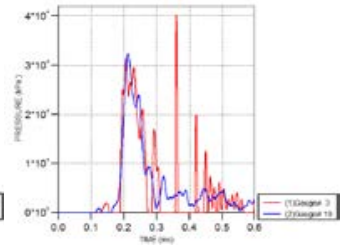

(b)

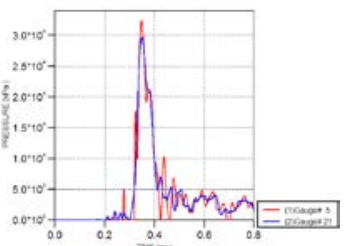

(d)

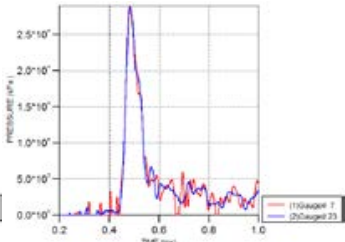

(f)

Figure 10. Comparison results of pressures on the center and circumference of the cross section of the water shock tube at the propagation distance of (a) $200 \mathrm{~mm}$, (b) $300 \mathrm{~mm}$, (c) $400 \mathrm{~mm}$, (d) 500 $\mathrm{mm}$, (e) $600 \mathrm{~mm}$ and (f) $700 \mathrm{~mm}$.

It can been seen from Figure 10 that when the propagation distance is less than $400 \mathrm{~mm}$, the pressure waveform on the center of the water shock tube is very chaotic. When the propagation distance is between $400 \mathrm{~mm}$ and $600 \mathrm{~mm}$, the peak pressures and the rising edge waveforms at the two monitor points are relatively close. When the propagation distance is greater than $600 \mathrm{~mm}$, the peak pressures at the two monitor points are the same, and the pressure waveforms are also basically consistent in most of the time. In summary, when the propagation distance is greater than 6 times the inner diameter, the plane wave is gradually formed. With the increase of the propagation distance, the plane wave is more and more stable.

\subsection{Similarity analysis of shock waves in infinite water area and water shock tube}

In order to dynamically calibrate the underwater shock wave pressure measurement system, it is required that the generated quasi- $\delta$ signal can simulate the shock wave pressure under the actual underwater working condition. The ratio of the length of the inner cavity to the inner diameter is defined as the L/D radio of the water shock tube. Keep the size of the inner diameter unchanged, and perform several groups of finite element simulation under the condition that the L/D ratio is $8,12,16$, 20, 24 and 28. Compare the quasi- $\delta$ signal obtained from the simulation with the explosion shock wave pressure waveform under the actual working conditions, and the results are shown in Figure 11. 


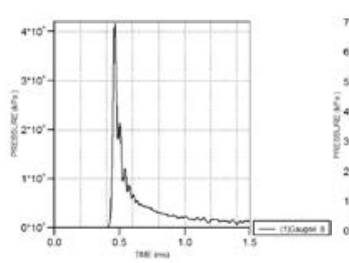

(a)

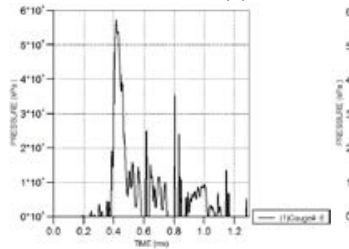

(c)

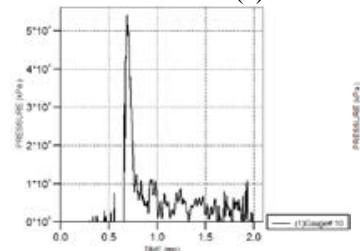

(e)

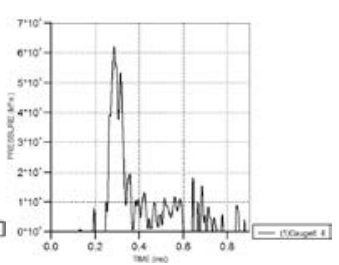

(b)

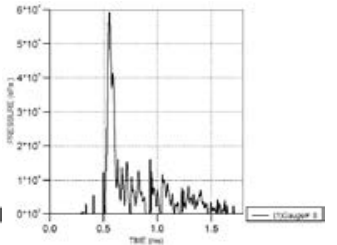

(d)

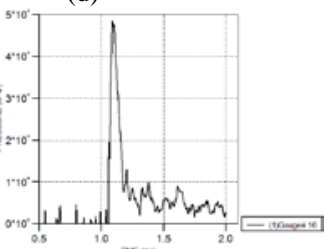

(f)

Figure 11. Comparison results between the quasi- $\delta$ signal obtained from the simulation and the explosion shock wave pressure waveform under the actual working conditions. (a) Shock wave pressure waveform at the propagation distance of $800 \mathrm{~mm}$ in the infinite water area. Shock wave pressure waveform in the water shock tube with the L/D ratio of (b) 8, (c) 12, (d) 16, (e) 20 and (f) 32.

As shown in the above figure, when the L/D ratio is 8 , the stable plane wave can not be formed. Therefore, the shock wave pressure waveform at the end face of the water shock tube is chaotic, which is far from the shock wave pressure in the infinite water area. When the L/D ratio is 12 , the rising edge of the shock wave pressure waveform at the end face is close to that in the infinite water area, but the wave oscillation is obvious in the subsequent attenuation stage. When the $\mathrm{L} / \mathrm{D}$ ratio is greater than 16, the shock wave pressure waveform at the end face is very close to that in the infinite water area in both the rising and attenuation stages. Therefore, to produce a quasi- $\delta$ signal highly similar to the shock wave pressures in the infinite water area, the L/D ratio of the designed water shock tube should be guaranteed to be no less than 16 .

\section{Conclusion}

Based on the Autodyn software, the propagation rule of the explosion shock wave in the infinite water area and the shock tube is studied in this paper. The simulation results in the infinite water area are in agreement with the calculation results of the classical empirical formula proposed by Cole, which shows that the finite element simulation is reliable. By analyzing the pressure waveforms on the center and circumference of the cross section of the water shock tube, the formation condition of the plane wave is obtained, that is, the propagation distance of the shock wave needs to be greater than 6 times the inner diameter of the water shock tube (the $\mathrm{L} / \mathrm{D}$ ratio is greater than 12). By changing the $\mathrm{L} / \mathrm{D}$ ratio of the water shock tube, multi groups of simulation are performed, and the simulation results are compared with the shock wave pressure waveforms in the infinite water area. According to the comparison results, it is found that the similarity between the quasi- $\delta$ signal in the water shock tube and the shock wave pressure under the actual underwater working condition improves with the increase of the $\mathrm{L} / \mathrm{D}$ ratio, and the quasi- $\delta$ pressure signal that meets the dynamic calibration requirements can be generated when the $\mathrm{L} / \mathrm{D}$ ratio is greater than 16 . According to the full text, the following conclusions can be obtained:

1 ) The water shock tube requires only a few mass of explosives to produce a quasi- $\delta$ signal with a larger peak pressure, which can effectively reduce the test cost.

2) The water shock tube used for the dynamic calibration of the underwater shock wave pressure 
measurement system should satisfy the basic condition that the L/D ratio is not less than 16 .

This paper has a certain reference significance for understanding the pressure propagation characteristics of the explosion shock wave in the water shock tube, and guiding the design of the shape size and the internal explosives of the water shock tube.

\section{Acknowledgment}

The authors would like to acknowledge support of the project by the National Defense Basic Scientific Research Project (No. JSJL20166060B001D).

\section{References}

[1] H. Wang, X. Zhu, Y. S. Cheng, and J. Liu, "Experimental and numerical investigation of ship structure subjected to close-in underwater shock wave and following gas bubble pulse,” Mar. Struct. Oxford, vol. 39, pp. 90-117, December 2014.

[2] X. Li, C. Zhang, X. Wang, and X. Hu, "Numerical study of underwater shock wave by a modified method of characteristics,” J. Appl. Phys. New York, vol. 115, pp. 0924-623, July 2014.

[3] R. Ghoshal, and N. Mitra, "Non-contact near-field underwater explosion induced shock-wave loading of submerged rigid structures: nonlinear compressibility effects in fluid structure interaction,” J. Appl. Phys. New York, vol.112, pp.287-303, July 2012.

[4] G. Wang, Y. Wang, W. Lu, W. Zhou, M. Chen, and P. Yan, "On the determination of the mesh size for numerical simulations of shock wave propagation in near field underwater explosion,” Appl. Ocean Res. Oxford, vol.59, pp.1-9, September 2016.

[5] L. Elkarous, C. Robbe and M. Pirlot, "Dynamic calibration of piezoelectric transducers for ballistic high-pressure measurement,” Int. J. Metrol. Qual. Eng. France, vol.7 pp.201, March 2016.

[6] A. Chang, X.Meng and B. Peng, "Analysis on Static and Quasi-static Sensitivity Characteristics of High-pressure Piezoelectric Sensors,” J. Ballistics Nanjing, vol. 26 pp.86-89, February 2014.

[7] "A guide for the dynamic calibration of pressure transducers," International Society of Automation, 2002.

[8] C. Matthews, F. Pennecchi and S. Eichstädt, "Mathematical modelling to support traceable dynamic calibration of pressure sensors,” Metrologia Bristol, vol.51 pp.326-338, May 2014.

[9] T. Gu, D. Kong and J. Jiang, "Pressure prediction model based on artificial neural network optimized by genetic algorithm and its application in quasi-static calibration of piezoelectric highpressure sensor,” Rev. Sci. Instrum. New York, vol.87 pp.125005, December 2016.

[10] W. Guo, T. C. Yu, and Z. L. Li, "Dynamic Calibration of Sensitivity of Shock Wave Pressure Sensor,” Chinese J. Explos. Propell. Xian, vol.29 pp.62-64, July 2006.

[11] M. W. Zhu, and X. J. Wen, "Water shock tube for high pressure dynamic calibration," Instrumentation and Measurement Technology Conference Hamamatsu, vol.2 pp.468-470, May 1994.

[12] C. K. Gong, and Y. X. Li, "Water shock tube of high pressure dynamic calibration,” Trans. Microsyst. Technol. Harbin, vol.27 pp.61-63, September 2008.

[13] M. Zhu, "The progress of dynamic calibration technology of high pressure measurement," J. Test Meas. Technol. Taiyuan, vol.9 pp.32-38, July 1995.

[14] W. Z. Chen, C. D. Xu, and D. R. Kong, "Study on Characteristics of Pulse Pressure Signal of Water Shock Tube,” J. Test Meas. Technol. Taiyuan, vol.31 pp.524-530, January 2017.

[15] R.H. Cole, “Underwater Explosions,” Dover Publications New York, 1948. 